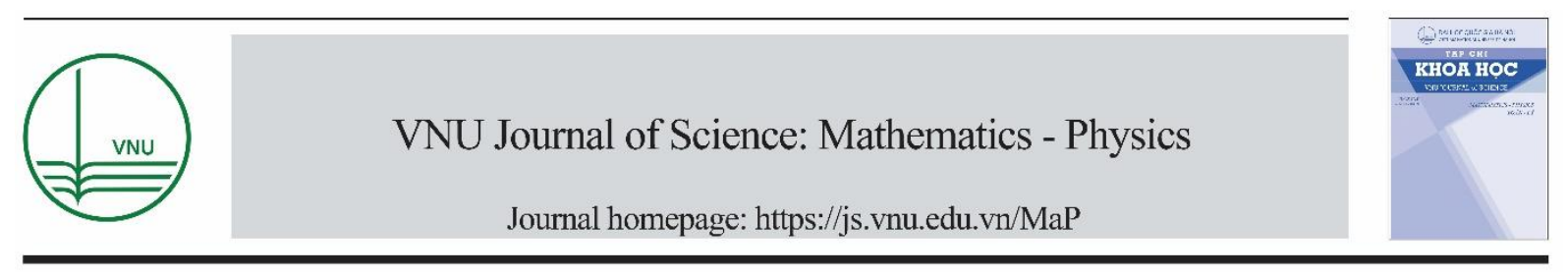

Original Article

\title{
Preparation and Microstructure of Acetate-based Lead-free BSZT Ferroelectric Thin Films Using Sol-gel Technique
}

\author{
Pham Thi Nguyet ${ }^{1}$, Nguyen Thi Minh Phuong ${ }^{1,2}$, Vu Thu Hien ${ }^{1, *}$, Vu Ngoc Hung ${ }^{1}$ \\ ${ }^{1}$ International Training Institute for Materials Science (ITIMS), \\ Hanoi University of Science and Technology, 1 Dai Co Viet, Hanoi, Vietnam \\ ${ }^{2}$ College of Industry and Trade, 1, Chua Cam, Trung Nhi, Phuc Yen, Vinh Phuc Vietnam
}

Received 15 January 2019

Revised 04 March 2019; Accepted 15 March 2019

\begin{abstract}
Ba}_{0.85} \mathrm{Sr}_{0.15}\right)\left(\mathrm{Ti}_{0.9} \mathrm{Zr}_{0.1}\right) \mathrm{O}_{3}$ (BSZT) lead-free ferroelectric thin films at the vicinity of the morphotropic phase boundary (MPB) were successfully deposited on $\mathrm{Pt} / \mathrm{Ti} / \mathrm{SiO}_{2} / \mathrm{Si}$ using a modified spin-coated sol-gel method. Microstructure and electrical properties of the thin film were studied. High-resolution synchrotron X-ray powder diffraction (SXRD) combined with Rietveld refinement revealed that the samples were crystallized in tetragonal perovskite structure with in-plane symmetry $(c<a)$. Raman spectra also confirmed a tetragonal perovskite crystalline lattice structure. Polarisation studies demonstrate that BSZT films exhibit a rather high saturation polarisation of $22.25 \mu \mathrm{C} . \mathrm{cm}^{-2}$. Leakage current behavior was obtained and possible conduction mechanism is discussed.
\end{abstract}

Keywords: xBST-(1-x)BZT ferroelectric materials, thin film technology, sol-gel.

\section{Introduction}

Ferroelectric materials have a unique combination of properties such as spontaneity, a switchable polarisation, piezoelectricity, and pyroelectricity. Therefore, such materials have been extensively studied for many applications, including non-volatile random access memories (NVRAMs), dynamic random access memories (DRAMs), high-frequency (GHz) bypass capacitors, infrared detectors, as well as tunable devices in microwave electronics $[1,2]$. Lead zirconate titanate PZT has been long time the

\footnotetext{
${ }^{*}$ Corresponding author.

E- mail address: hien.vuthu@ hust.edu.vn

https//doi.org/ 10.25073/2588-1124/vnumap.4316
} 
leading commercial material for actuators, sensors, and transducer devices due to its excellent piezoelectric properties in the vicinity of the morphotropic phase boundary (MPB) between the rhombohedral and tetragonal phases [3]. However, the wide utilization of PZT has increased serious environmental and health concerns due to the toxicity of lead element during the manufacture, and recycling of these devices. Therefore, it is highly desirable to search for lead-free alternative materials for these applications [4]. In the recent years, there have been surged to study and develop lead-free ferroelectric ceramics, which include $\mathrm{BaTiO}_{3}-\mathrm{BTO}, \mathrm{BiFeO}_{3}(\mathrm{BFO}) \mathrm{Na}_{0.5} \mathrm{Bi}_{0.5} \mathrm{TiO}_{3}-\mathrm{K}_{0.5} \mathrm{Bi}_{0.5} \mathrm{TiO}_{3}$ (NKBT), $\mathrm{Na}_{0.5} \mathrm{~K}_{0.5} \mathrm{NbO}_{3}(\mathrm{KNN})$, etc [5]. However, the main drawback of these materials is the presence of highly volatile elements, such as $\mathrm{Na}, \mathrm{Bi}$ and $\mathrm{K}$. In consequence, those materials are often difficult to prepare in the precise stoichiometry. It then makes their ferroelectricity or piezoelectricity is inferior to PZT.

Much previous research show that by suitable doping engineering both at $\mathrm{Ba}^{2+}$ or/and $\mathrm{Ti}^{4+}$ sites in BTO with either equipollent or aliovalent metals, one can enhance significantly the dielectric, ferroelectric and piezoelectric properties. The strong improvement in piezoelectric effect is attributed to a phase coexistence (rhombohedral and tetragonal) MPB composition starting at the triple point (tetragonal-cubic- rhombohedral), which results in a very low energy barrier for polarisation rotation and lattice distortion [6].This research led to worldwide attention on lead-free $(1-\mathrm{x})\left[\mathrm{Ba}\left(\mathrm{Zr}_{0.2} \mathrm{Ti}_{0.8}\right) \mathrm{O}_{3}-\right.$ $\left.\mathrm{x}\left(\mathrm{Ba}_{0.3} \mathrm{Ca}_{0.7}\right) \mathrm{TiO}_{3}\right]$ ceramics for various applications.

In this work, we report the preparation of $\left(\mathrm{Ba}_{0.85} \mathrm{Sr}_{0.15}\right)\left(\mathrm{Zr}_{0.1} \mathrm{Ti}_{0.9}\right) \mathrm{O}_{3}-(\mathrm{BSZT})$ sample via sol-gel route, their microstructure and electrical properties. BSZT thin films $(\sim 225 \mathrm{~nm})$ were deposited on conductive $\mathrm{Pt} / \mathrm{Ti} / \mathrm{SiO}_{2} / \mathrm{Si}$ substrates followed by annealing at $650{ }^{\circ} \mathrm{C}$. The crystalline structure (with its space group), room-temperature hysteresis ( $\mathrm{P}-\mathrm{E})$ measurements and leakage current behavior of these films were studied.

\section{Experiments}

\subsection{BSZT Sol preparation}

Barium acetate $\mathrm{Ba}\left(\mathrm{CH}_{3} \mathrm{COO}\right)_{2}$, strontium acetate $\mathrm{Sr}\left(\mathrm{CH}_{3} \mathrm{COO}\right)_{2}$, zirconium n-propoxide $\mathrm{Zr}\left(\mathrm{OC}_{3} \mathrm{H}_{7}\right)_{4}$ and isopropoxide titanate $\mathrm{Ti}\left(\mathrm{OC}_{3} \mathrm{H}_{7}\right)_{4}$ were used as starting materials. Acetic acid $\mathrm{CH}_{3} \mathrm{COOH}$ and 2-methoxyethanol $\mathrm{CH}_{3} \mathrm{OCH}_{2} \mathrm{CH}_{2} \mathrm{OH}$ were selected as solvents, formamide $\mathrm{NH}_{2} \mathrm{CHO}$ as additives and acetylacetone $\mathrm{CH}_{3} \mathrm{COCH}_{2} \mathrm{COCH}_{3}$ as a reagent to stabilize the solution.

First, barium acetate and strontium acetate $99.95 \%$ (p.a Sigma Aldrich) were dissolved in glacial acetic acid and 2-methoxylethanol at $120{ }^{\circ} \mathrm{C}$ for $4 \mathrm{~h}$. Acetylacetone was used to stabilize isopropoxide titanate and zirconium n-propoxide, and then the modified precursor was added to the above solution with constant rate stirring at room temperature. To adjust viscosity, surface tension of the precursor, formamide was added to the solution under ultrasonic agitation. $\mathrm{pH}$ value was adjusted using glacial acetic acid to remain 2-4. The resultant solution was filtered to form the stock solution. The solution was yellow, clear and transparent and stabilize for several months.

\section{BSZT thin film depostion characterization}

The above mentioned solution was then spin-coated on the $\mathrm{Pt} / \mathrm{Ti} / \mathrm{SiO}_{2} / \mathrm{Si}$ substrates at $3000 \mathrm{rpm}$ for $30 \mathrm{~s}$. The as-deposited BSZT thin films were heated by a four-step heating procedure: first heated at a low temperature of $150{ }^{\circ} \mathrm{C}$ for $10 \mathrm{~min}$ to dry the gel; then pyrolyzed at $350^{\circ} \mathrm{C}$ in air for $15 \mathrm{~min}$; the films were crystallized at about $650{ }^{\circ} \mathrm{C}$ by rapid annealing (flowing rate was $50{ }^{\circ} \mathrm{C} / \mathrm{min}$ ) for $30 \mathrm{~min}$. To gain the crack-free films, an additional pre-annealing was used at $550{ }^{\circ} \mathrm{C}$ before rapid thermal annealing. The 
additional step is useful to eliminate the crack phenomenon owing to the shrinkage of thin films fabricated by the sol-gel.

\subsection{Microstructural and electrical measurements}

Characterization of the crystalline structure of BSZT powders was carried out using Synchrotron Xray diffraction (SXRD) at Beamline SAXS of the Synchrotron Light Research Institute (Thailand). A data processing software SAXSIT was used to process the 2D scattering patterns for further analyses. The diffraction data were analyzed using Rietveld method with the help of FullProf program [7].The diffraction peaks were modeled by pseudo-Voigt function. A standard of $\mathrm{LaB}_{6}$ was used to determine instrument broadening. The refinement fitting quality was checked by goodness of fit $\left(\chi^{2}\right)$ and weighted profile R-factor $\left(\mathrm{R}_{\mathrm{wp}}\right)$. The calculated results are accepted with $\chi^{2}$ should approach 1 and $\mathrm{R}_{\mathrm{wp}}$ must be close to or less than $10 \%$. The grain size and morphology were investigated by scanning electron microscopy (SEM) using a Nova NanoLab 600 microscope at $15 \mathrm{kV}$. The phase compositions and their relative stabilities in these BSZT thin film were carried out by Raman scattering experiments using a micro-Raman spectrometer (Jobin-Yvon LabRAM HR 800 UV).

For electrical measurements, capacitors with a metal-insulator-metal (MIM) structure were fabricated. Circular Pt top electrodes (100 nm thick) were deposited with a shadow mask $\sim 100 \mu \mathrm{m}$ in diameter and conductive Pt substrates acted as the bottom electrode. Ferroelectric properties and leakage current were obtained at $1 \mathrm{kHz}$ with a TF Analyzer 2000E (aixACCT Systems GmbH, Germany).

\section{Results and discussion}

BSZT samples obtained to possess a polycrystalline perovskite crystal structure and exhibit a tetragonal phase (space group Pmm4) after annealing at $650{ }^{\circ} \mathrm{C}$ for $30 \mathrm{~min}$. Those features are characterized by the main $(001) /(100) ;(110) /(101) ;(111)$ and $(200) /(002)$ peaks at approximately $2 \theta-$ $22^{\circ} ; 31.4^{\circ} ; 38.6^{\circ}$ and $45.2^{\circ}$, respectively (Figure 1 ). The Rietveld refinement results indicated that the tetragonal perovskite structure with atoms in positions $\mathrm{Ba}$ in $1 \mathrm{a}(0,0,0)$, Ti in $1 \mathrm{~b}(1 / 2,1 / 2, \mathrm{z})$, oxygen in $1 \mathrm{~b}(1 / 2,1 / 2,-0.0153)$ and in $2 \mathrm{c}(1 / 2,0,0.513)$. These obtained results are in good agreement with previous studies on crystal structure of nanoscale $\mathrm{BaTiO}_{3}[8,9]$.

The microstructural parameters (the crystallite size $\mathrm{D}$ and lattice microstrain $\Delta \mathrm{d} / \mathrm{d}$ (where $\mathrm{d}$ is interplanar distance) were obtained by analysis of the peak broadening on applying Rietveld method using FullProf program with the instrumental resolution function identified by SXRD analysis of the $\mathrm{LaB}_{6}$ standard. The refined values of structural parameters including lattice constant (a, c), unit cell volume $(\mathrm{V}), \mathrm{z}(\mathrm{Ti})$, the crystallite size (D), microstrain $\Delta \mathrm{d} / \mathrm{d}$ and fitting quality $\left(\chi^{2}\right.$ and $\left.\mathrm{R}_{\mathrm{wp}}\right)$ are given in Table 1. As seen, substitution of smaller $\mathrm{Sr}^{2+}$ ion $\left(\mathrm{rSr}^{2+}=1.58 \AA\right)$ for $\mathrm{Ba}^{2+}\left(\mathrm{rBa}^{2+}=1.75 \AA\right)$ leads to a decrease of lattice parameters and unit cell volume. In addition, the substitution also induces a more disordered structure shown by the increase of microstrain value.

Table 1. Structural parameters of the samples estimated from Rietveld refinement. Statistical errors are indicated in the last significant digit

\begin{tabular}{lllllllll}
\hline Sample & $\mathrm{a}$ & $\mathrm{c}$ & $V, \AA^{3}$ & $D, \mathrm{~nm}$ & $z(\mathrm{Ti})$ & $\begin{array}{l}\Delta \mathrm{d} / \mathrm{d}, \\
\%\end{array}$ & $\begin{array}{l}\chi^{2} \\
R_{\mathrm{wp}},\end{array}$ \\
\hline BSZT & $4.0294(5)$ & $4.0082(9)$ & $65.08(2)$ & $22.7(2)$ & $0.4825(5)$ & $0.77(1)$ & 1.39 & 9.15 \\
BTO & $3.9940(8)$ & $4.0124(9)$ & $64.01(3)$ & $23.2(1)$ & $0.484(8)$ & $0.48(1)$ & 1.33 & 7.70 \\
{$[10]$} & & & & & & & & \\
\hline
\end{tabular}




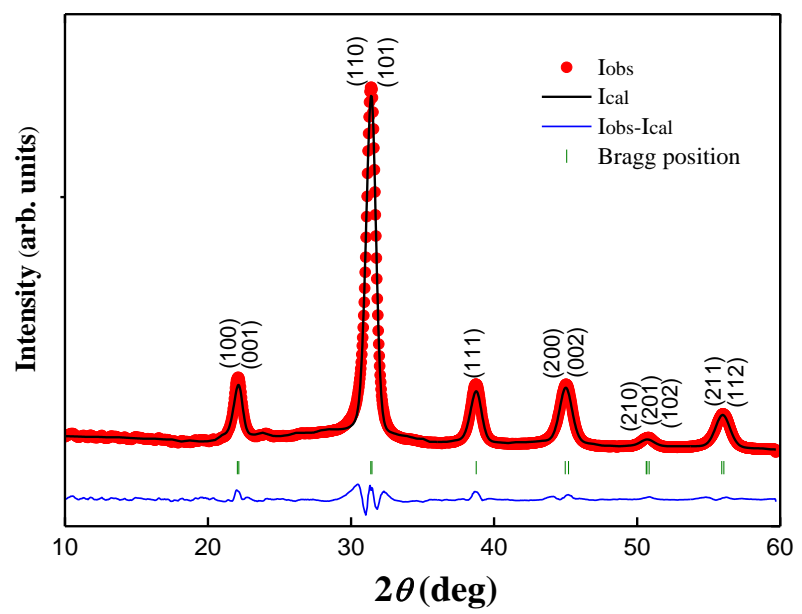

Figure 1. SXRD diffraction pattern of the BSZT sample and processed by the Rietveld method. The Miller indices of the main phase peaks and the peak positions are indicated.

In order to confirm the structure changes of BSZT sample with doped amount of $\mathrm{Sr}$ and $\mathrm{Zr}$, Raman scattering spectra were measured. Typical room temperature Raman spectra of BSZT thin film is shown in Figure 2. Some regions of the obtained Raman signal were rather noisy. However, one can observe four disorder-activated bands centered at $\sim 137.8,302,517.3$ and $742 \mathrm{~cm}^{-1}$ (marked as A, B, C, and D, respectively, in Fig. 2). Their activation is expected from the eight-site model due to disorder of Ti ions, which can occupy four off-center sites in the tetragonal phase.

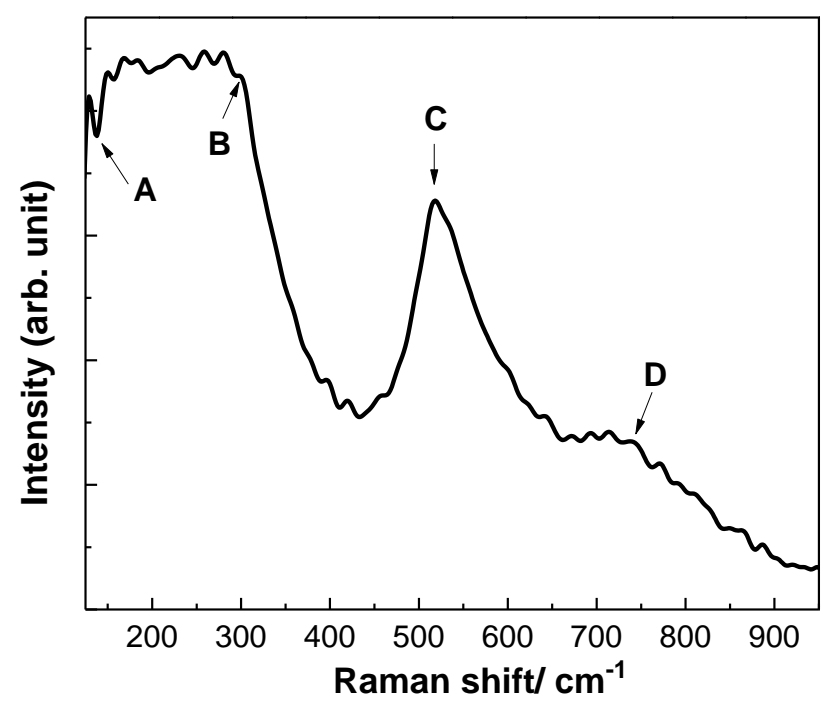

Figure 2. Room temperature Raman spectra of BSZT thin films annealing at $650{ }^{\circ} \mathrm{C}$

Apparently, the incorporation of $\mathrm{Zr}$ and $\mathrm{Sr}$ atoms causes local distortions and breaks partially the translational symmetry of the BTO lattice and, therefore, disorder-activated background is much more 
complicated in BSZT [11]. The disappearance of the well-known dip at $178 \mathrm{~cm}^{-1}$ and the insignificant $\mathrm{A} 1+\mathrm{E}(\mathrm{TO}+\mathrm{LO})$ peak at $301 \mathrm{~cm}^{-1}$ is characteristic of the cubic-to tetragonal phase transition [12].

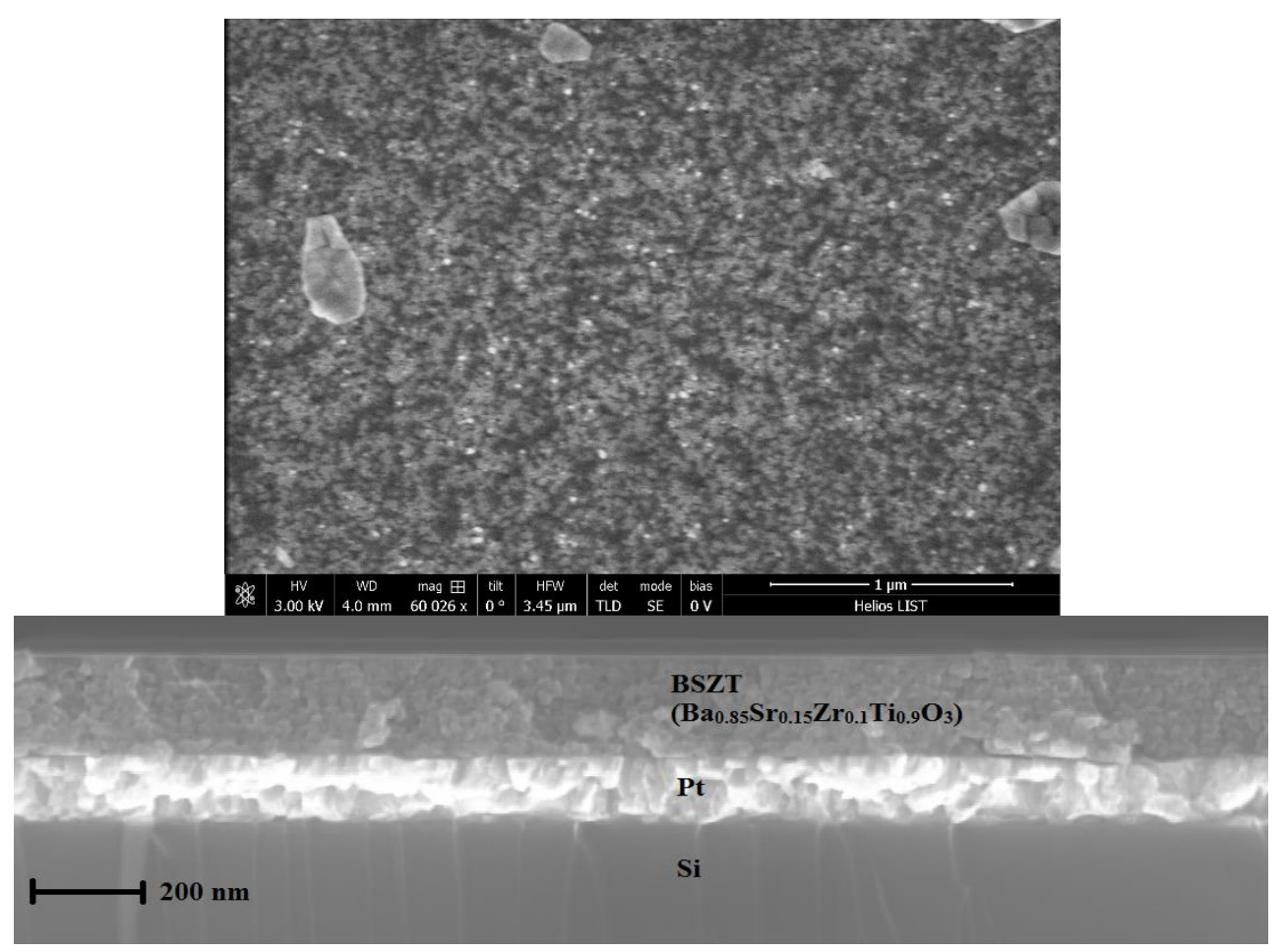

Figure 3. SEM images of the top surface (upper part) and cross-section of sol-gel BSZT thin films (lower part).

Figure 3 presents the SEM images of the surface of the BSZT films annealed at $650{ }^{\circ} \mathrm{C}$. The grain size is around $30 \mathrm{~nm}$ and the majority of the grains are separated by porosity which is darker surrounding regions. An increase of annealing temperature may allow the coalescence of those small grains. The coalescence also leads to increase in the size of the intergranular porosity. Surface mappings were then confirmed by cross-section analysis shown in the lower image. As seen, the out-of-plane grain sizes and morphologies are approximately the same than the top surface: the grains have a spherical shape and are disordered in arranging. The obtained thickness of the film is ca. $225 \mathrm{~nm}$ for 8 coating layers which turn out $28 \mathrm{~nm}$ per coating layer.

The ferroelectric nature of prepared BSZT thin films was confirmed by a hysteresis loop of polarisation (P) as a function of an applied electric field (E) at $1 \mathrm{kHz}$, as shown in Figure 4. Careful examination of different $\mathrm{Pt}$ top electrodes on shadow masks with the Pt bottom electrode on the Si substrate at room temperature revealed saturated ferroelectric hysteresis loops. The hysteresis loops indicate that as the voltage increases, the $P-E$ loop becomes slanted. As the electric field increased from $0.5 \mathrm{kV} / \mathrm{cm}$ to $350 \mathrm{kV} / \mathrm{cm}$, both saturation polarisation $\left(P_{\mathrm{s}}\right)$ and the remnant polarisation $\left(P_{\mathrm{r}}\right)$ were increased. Ferroelectric loops showed a maximum of $22.27 \mu \mathrm{C} / \mathrm{cm}^{2}$ saturation polarisation and $5.85 \mu \mathrm{C} / \mathrm{cm}^{2}$ remnant polarisation in the BSZT thin films. These obtained values are smaller than that of the bulk ceramic counterpart [13]. The much higher coercive feld $\left(\mathrm{E}_{\mathrm{c}} \sim 66.5 \mathrm{kV} / \mathrm{cm}\right)$ might be owing to much smaller grains in the BSZT films in comparison with the bulk ceramic hence there being many more grain boundaries. Besides, it might also be due to substrate clamping effects [14]. 


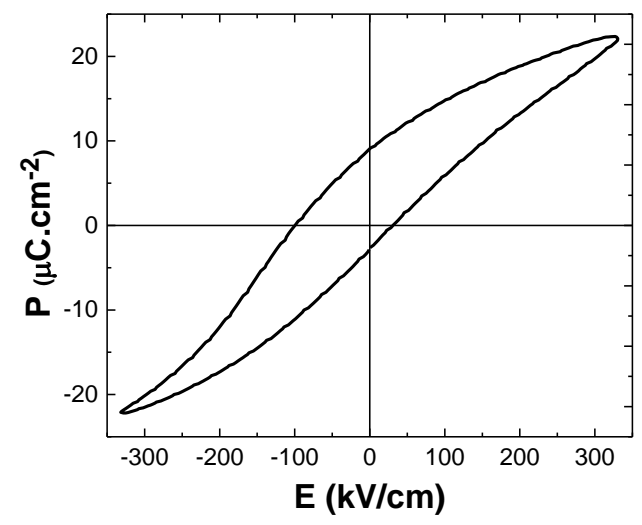

Figure 4. P-E loop of the BSZT film at $1 \mathrm{kHz}$.

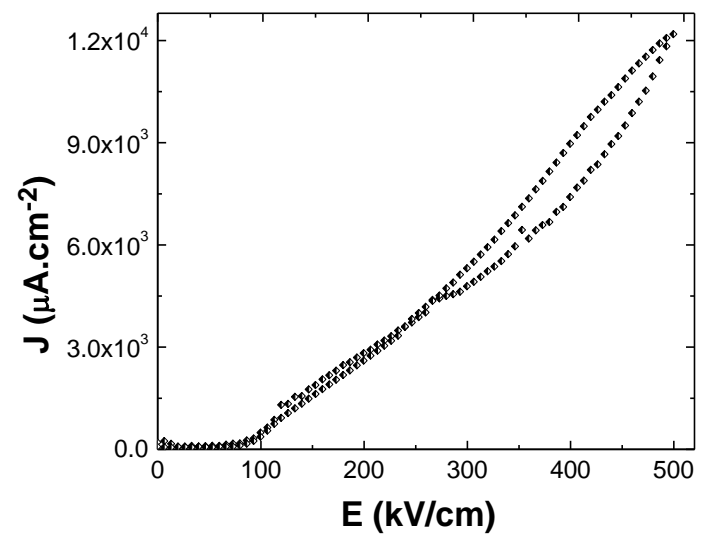

Figure 5. Current density as a function of applied electric field (J-E) for BSZT thin film.

In order to determine the conduction mechanism of the BZT-BST thin film, measurements of its current density-electric field (J-E) characteristics were performed. It was seen that the conduction through the dielectric BSZT film is noticeable when a relatively large bias voltage is applied. Of all the possible mechanisms, this conduction current shows a non-Ohmic behavior in the electric field region below $100 \mathrm{kV} / \mathrm{cm}$ as is illustrated in Figure 5. However, we found a steady increase of the leakage current when the electric field was increased (above $100 \mathrm{kV} / \mathrm{cm}$ ). It is probably bulk-limited conduction mechanism. Investigations of the leakage current-temperature dependence of the thin films is in progress. These studies may help to elucidate the conducting mechanism across the metal-dielectric junction.

\section{Conclusion}

In summary, we have successfully synthesized BSZT phase pure powder and deposited BSZT thin films on $\mathrm{Pt} / \mathrm{Ti} / \mathrm{SiO}_{2} / \mathrm{Si}(100)$ substrates by the sol-gel process. The additional formamide and acetylacetone as an additive and stabilizer is useful to eliminate the effect of the films bulk shrinkage. The perovskite phase of thin films was formed at $650{ }^{\circ} \mathrm{C}$. The thin films were smooth and crack-free. BSZT films exhibited ferroelectric properties with rather high saturated polarization, $\mathrm{P}_{\mathrm{s}}$ of 22.25 $\mu \mathrm{C} / \mathrm{cm}^{2}$, and coercive field $\left(\mathrm{E}_{\mathrm{c}} \sim 66.5 \mathrm{kV} / \mathrm{cm}\right)$.

\section{Acknowledgments}

The authors acknowledge the financial support of the National Foundation for Science \& Technology Development of Vietnam (Nafosted, Project Grant 103.02-2017-14).

\section{References}

[1] J.F. Scott, Applications of Modern Ferroelectrics, Science 315 (2007) 954-959. 
[2] M. Bibes, V. Garcia, S. Fusil, K. Bouzehouane, N.D. Mathur, A. Barthe, Giant tunnel electroresistance for nondestructive readout of ferroelectric states, Nature 460 (2009) 2-5.

[3] G.H. Haertling, Ferroelectric Ceramics: History and Technology, J. Am. Ceram. Soc. 82 (1999) 797-818.

[4] P.K. Panda, B. Sahoo, PZT to Lead Free Piezo Ceramics: A Review, Ferroelectrics. 474 (2017) 128-143.

[5] I. Coondoo, N. Panwar, A. Kholkin, Lead-free piezoelectrics: Current status and perspectives, J. Adv. Dielect. 3 (2013) 1330002.

[6] W. Liu, X. Ren, Large Piezoelectric Effect in Pb-Free Ceramics, Phys. Rev. Lett. 103 (2009) 257602.

[7] L.B. Mccusker, R.B. Von Dreele, D.E. Cox, D. Loue, P. Scardi, Rietveld refinement guidelines, J. Appl. Cryst. 32 (1999) 36-50.

[8] C.J. Xiao, C.Q. Jin, X.H. Wang, Crystal structure of dense nanocrystalline $\mathrm{BaTiO}_{3}$ ceramics, Mater. Chem. Phys. 111 (2008) 209-212.

[9] M.B. Smith, K. Page, T. Siegrist, P.L. Redmond, E.C. Walter, R. Seshadri, et al., Crystal Structure and the Paraelectric-to-Ferroelectric Phase Transition of Nanoscale $\mathrm{BaTiO}_{3}$, J. Am. Chem. Soc. 130 (2008) 6955-6963.

[10] T.H. Vu, T.L. To, D.M. Nguyen, N.H. Vu, Cau truc pha va dac trung sat dien cua mang mong $\mathrm{Ba}\left(\mathrm{Ti}_{0.9} \mathrm{Zr}_{0.1}\right) \mathrm{O}_{3}$ che tao bang phuong phap Sol-gel, SPMS Conf. 2 (2017) 523-526.

[11] L.H. Robins, D.L. Kaiser, L.D. Rotter, P.K. Schenck, G.T. Stauf, L.H. Robins, et al., Investigation of the structure of barium titanate thin films by Raman spectroscopy, J. Appl. Phys. 76 (1994) 7487-7498.

[12] R. Farhi, M. El Marssi, A. Simon, J. Ravez, A Raman and dielectric study of ferroelectric $\mathrm{Ba}\left(\mathrm{Ti}_{1-\mathrm{x}} \mathrm{Zr}_{\mathrm{x}}\right) \mathrm{O}_{3}$ ceramics, Eur. Phys. J. B. 9 (1999) 599-604.

[13] Y. Li, H. Cheng, H. Xu, Y. Zhang, P. Yan, T. Huang, Electromechanical properties of (Ba,Sr)(Zr,Ti) $\mathrm{O}_{3}$ ceramics, Ceram. Int. 42 (2016) 10191-10196.

[14] V.P. Sreenivas, K.P. Dhiren, B.C. Douglas, M. Tomozawa, G.L. Sharma, J.F. Scott, et al., Structure, dielectric, ferroelectric, and energy density properties of $(1-\mathrm{x}) \mathrm{BZT}-\mathrm{xBCT}$ ceramic capacitors for energy storage applications, J. Mater. Sci. 48 (2013) 2151-2157. 